ORIGINAL ARTICLE: EPIDEMIOLOGY, CLINICAL PRACTICE AND HEALTH

\title{
Perceived disability from hearing and voice changes in the elderly
}

\author{
Simonetta Monini, ${ }^{1}$ Chiara Filippi, ${ }^{1}$ Rossella Baldini ${ }^{2}$ and Maurizio Barbara ${ }^{1}$ \\ ${ }^{1}$ Department of Neuroscience, Mental Health and Sensory Organs, and ${ }^{2}$ Department of Anatomical, Histological, Medico-legal and \\ Locomotor Sciences, Sapienza University, Rome, Italy
}

\begin{abstract}
Aim: Dysphonia and hearing loss are underestimated conditions in the elderly, despite their significant prevalence (18\% and $50 \%$, respectively) and their sociopsychological implications. Previous studies have shown that the reason for this lack of consideration is related to the general misconception of a simple age-related issue, as well as to the reduced communication requirements of this population, which can result in infrequent requests/supply of care. The purpose of the present study was to evaluate, within an elderly population, the subjective perception of hearing and voice dysfunctions, the resulting changes in communication skills, and the perception of handicap and disability.
\end{abstract}

Methods: Four anonymous questionnaires were administered to 400 participants (218 men, 182 women) aged older than 65 years, some of whom (276) were hospitalized and some of whom (124) were outpatients. The questionnaires consisted of questions regarding age-related changes in voice, multiple-choice questions on the qualitative characteristics of the voice, questions regarding verbo-acoustic communication (hearing), the Voice Handicap Index, and the Self Assessment of Communication regarding the perception of hearing loss-related handicap and disability. Statistical correlations were calculated for voice dysfunction between the perception of disability and the clinical assessment of voice quality obtained by the Grade, Roughness, Breathiness, Asthenia, Strain scale, and between the perception of disability and the demand for care.

Results: More than half of the elderly patients reported not perceiving voice changes throughout their lives. Most of the participants were satisfied with their own voices, although $65 \%$ of them judged them to be qualitatively altered, and in $31.5 \%$ of the participants, pathology was found on phoniatric evaluation. Low scores for vocal handicap (Voice Handicap Index) were found, and the type of perceived disability was mainly physical, although the association between Voice Handicap Index scores and Grade, Roughness, Breathiness, Asthenia, Strain was statistically significant. A total of $62 \%$ of the patients perceived hearing changes over their lifetimes not related to previous ear infections, but significantly correlated with a family history of hearing problems and with the need for specialist consultations. However, the perception of hearing loss handicaps and disability showed lower mean values, showing that older patients recognized dysfunction, but did not consider it to be a disability.

Conclusions: The present study showed that, despite the relevant incidence of hearing and voice disorders among the elderly population, the implications for communication abilities seems to be underestimated. Hence, it appears to be extremely important to undergo specialist screening consultations to detect eventual voice and hearing alterations,

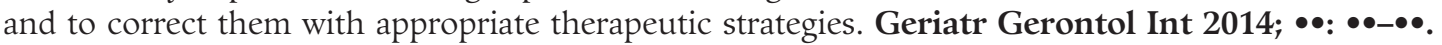

Keywords: elderly, hearing handicap inventory, presbycusis, presbyphonia, Voice Handicap Index.

Accepted for publication 14 December 2013.

Correspondence: Professor Maurizio Barbara MD PhD, NESMOS Department, Medicine and Psychology, Sapienza Rome 00189, Italy. Email: maurizio.barbara@uniroma1.it

\section{Introduction}

Aging is a physiological process that unavoidably affects every individual in his/her lifetime. When the individual reaches this condition, a series of physical, social, affective and psychological changes occur, possibly modifying the quality of life. The remarkable increase in elderly people, which in Western countries has been related to 
both increased longevity and decreased birth numbers, ${ }^{1}$ has shown the need for better approaches to solving the different problems that arise in this elderly population. One of the major issues certainly regards communication, which, apart from modern technological tools (Internet, texting) that are still uncommonly used by the elderly population, strongly relies on auditory and voice performance. To our knowledge, age-related modifications; that is, presbycusis and presbyphony, have not been adequately investigated so far in terms of their real incidences and patients' subjective perceptions. ${ }^{1}$ In fact, in previous studies, which are often difficult to compare because of the different time intervals considered, a mean prevalence between $12 \%$ and $20 \%$ of dysphonia has been reported, being functional in origin in $5 \%$ of cases. ${ }^{2}$ As far as hearing is concerned, data from the World Health Organization (WHO) show that approximately 500 million elderly individuals present with a hearing impairment, and that in $10 \%$ of the cases, it is sufficiently relevant to compromise communication abilities. This percentage approaches $40 \%$ in more elderly subjects, specifically to $49 \%$ between 70 and 80 years-of-age, in accordance with more than $80 \%$ of hearing deficits occurring in the elderly (National Center for Health Statistics, 1994). ${ }^{3}$ Nonetheless, rehabilitative procedures do not represent a common solution if one considers that in Italy, for instance, $63 \%$ of the hearing-impaired population, that is, $12 \%$ of the whole population (7 million individuals), is aged older than 65 years, but just $16 \%$ of these people $(720000)$ routinely own or utilize a hearing aid.

In general, it would seem that the majority of the elderly population erroneously accepts both hearing loss and voice changes as inevitable age-related issues, and they are led to underestimate these issues' severity, presumably because of their minor social impact on the conditions of pensioners. ${ }^{3-5}$ Therefore, precise data on the real incidences of phonatory and auditory dysfunctions in the elderly population are difficult to obtain, unless specialist consultations are systematically undertaken and are correlated with subjective perception of the disability, in terms of psychological, social and emotional aspects. ${ }^{5}$

For this purpose, specific questionnaires can be utilized in clinical practice: the Self Assessment of Communication (or SAC) ${ }^{6}$ for subjective evaluation of hearing; the Voice Handicap Index (or VHI) ${ }^{7}$ for voice assessment; and the Hearing Handicap InventoryElderly Screening (or HHI-ES) ${ }^{8,9}$ for impact on quality of life. Previous studies have shown that, even with negative effects on quality of life, the general health condition might not be affected. ${ }^{10-13}$

The present study was designed to shed some light on the subject, through the quantification, by a singlecenter, observational, analytic survey, of the real subjective perception of eventual phonatory and auditory dysfunctions in an elderly population, as well as its correlation with objective specialist evaluations.

\section{Methods}

A total of 400 participants, aged older than 65 years, were observed at a tertiary university hospital during the period of January-July 2012 and were divided in two groups: (i) group A included 276 participants (169 women, 107 men) who had been hospitalized (H) in non-otorhinolaryngological wards; and (ii) group B included 124 participants (49 men, 75 women) who underwent otorhinolaryngological consultations as outpatients $(\mathrm{O})$ for problems not related to voice or hearing.

The present study received the approval of the local ethical committee as an observational study, in agreement with privacy regulations, because it was accomplished by the administration of anonymous questionnaires.

The criteria for being included in the present study were fully collaborative behavior, the absence of known laryngeal or auricular organic pathologies, as well as of voice and hearing changes, and the absence of neurological, psychiatric or severe cardiovascular diseases.

The study protocol included the administration of anonymous questionnaires. First, the patients were asked to sign an informed consent form on completion of the study (Appendix S1), and general health conditions were also collected (Appendix S2). Then, the following four questionnaires were administered:

1 Subjective anonymous questionnaire on voice modifications in the elderly (Appendix S3), consisting of:

A six questions on voice modification, with yes/no answers; and

B multiple-choice questions on qualitative changes in the acoustic characteristics of the voice, regarding intonation, intensity and tone.

2 Subjective anonymous questionnaire on hearing function (Appendix S4), consisting of nine questions, with yes/no answers, on the presence of eventual hearing disorders, family history of deafness, prior audiological consultations or requests to undergo such a consultation.

3 Voice Handicap Index (VHI), short version (SF-30), subdivided in three subscales, Physical (P), Functional $(\mathrm{F})$ and Emotional $(\mathrm{E})$, each with the same number of questions. Subscale $\mathrm{P}$ investigated the patient's perception of laryngeal or speech impairments; subscale F investigated the impact of speech impairment on daily activities; and subscale E investigated the patient's affective reaction to phonatory impairment. ${ }^{7}$ The questionnaire utilized commonly used statements for describing one's own voice and its effects on daily life. The patient was asked to cross-highlight those statements that better described the experiences of daily life, using adverbial terms 
including "never", "almost never", "sometimes", "almost always" and "always". The score was 0 for "never" and 4 for "always". The final evaluation of the results considered the partial scores on the three subscales and the total score, which ranged from 0 to 120 , the latter representing the maximally perceived phonatory handicap (Appendix S5).

4 Anonymous questionnaire on the subjective perception of verbal-acoustic communication (Self Assessment of Communication [SAC]). ${ }^{6}$ The SAC is a multiple-choice questionnaire divided in two sections: the first consisting of four questions that evaluate the subjective handicap perception; and the second consisting of five questions on disability perception relative to the hearing impairment. The answers were related to the frequency of occurrence of what was asked ("almost never or never", "occasionally", "half of the time", "frequently" and "often"). The scores ranged from 0 to 36 , with 0 indicating "almost never or never" and 4 indicating "always". Separate calculations were carried out regarding handicap, disability and the overall score (Appendix S6).

An objective evaluation was undertaken only for voice disturbances, to correlate the objective perceived scale of impairment to the subjective scale. For this purpose, the Grade, Roughness, Breathiness, Asthenia, Strain (GRBAS) scale, ${ }^{14}$ which is a clinical perceptive-auditory scale used to characterize dysphonic voices, was used by utilizing internationally standardized perceptive parameters, such as hoarseness, force, asthenia and whistling, along with a general $(\mathrm{G})$ evaluation of severity that was carried out by a specialized professional, namely a phoniatrician (Appendix S7).

For each task, the evaluation considered groups A (hospitalized) and B (outpatients) separately, as well as total population, and men and women.

The characteristics of the enrolled individuals were described by common frequency tables for the categorical variables and by the calculation of synthetic indices, such as means, standard deviations, medians and other quantiles, for the continuous variables.

Differences among the groups were analyzed with non-parametric tests $\left(\chi^{2}\right.$-test and Fisher test) for categorical variables, and with point-biserial correlations for continuous and dichotomous variables. Significance was considered when $P<0.05$. STATA software version 9.0 (StataCorp LP, College Station, TX, USA) was used for all the analyses. For questionnaires 1 and 2, the percentages of positive and negative answers were calculated for all the questions, except for question no. 4, for which the numbers of patients with perceived altered voices were counted, as well as the incidences of single dimensions relative to the perception of altered tone. In the VHI questionnaire, the total score and the partial scores for subscales P, E and F were evaluated. For the SAC questionnaire, the evaluation included the total score and, separately, the scores obtained for the groups of questions for handicap evaluation and for disability evaluation. The following other correlations were carried out:

1 For questionnaire I (Appendix S3):

- between positive answers to questions 1 (Did your voice change during your life?) and 2 (Did your voice change during recent years?) and those to questions 5 (Did you undergo a consultation for your voice?) and 7 (Do you think a specialist consultation for your voice is necessary?);

- between positive answers to question 3 (Do you like your present voice?) and the objective presence of tone alterations (GRBAS-positive); and

- among patients who signaled a tone voice alteration to question 4 (Would you consider a change in your voice tone?) and the percentage of those who showed clinically evident (GRBAS scale) voice tone alterations; and

2 For questionnaire II:

- between positive answers to questions 1 (Did your hearing change during your life?), 2 (Has your hearing changed during recent years?), 8 (Do you have noises in your ears?) and 9 (Do you suffer from vertigo?) with positive answers to questions 3 (Did you undergo an audiological test?), 4 (Have you been examined by an audiologist?), 5 (Do you think you are in need of an audiological examination for your hearing?), 6 (Are there cases of hearing loss in your family?) and 7 (Did you ever suffer an ear infection?);

- between the VHI score and question 7 of questionnaire 1 (Do you think a specialist consultation for your voice is necessary?);

- between VHI score and the objective evaluation with the GRBAS scale; and

- between SAC score and question 5 of questionnaire 2 (Do you think you are in need of an audiological examination for your hearing?).

\section{Results}

\section{Subjective and objective voice evaluation}

\section{Questionnaire Voice Quality}

Question no. 1 (Did your voice change during your life?) A total of $46 \%$ of hospitalized ( $\mathrm{H}$, group A) patients answered positively and $54 \%$ answered negatively; $56.4 \%$ of the outpatients $(\mathrm{O}$, group $\mathrm{B})$ answered positively and $44 \%$ of them answered negatively. Overall, the positive and negative answers were 50/50.

Questions no. 2 (Did your voice change during recent years?) A total of $36 \%$ of the $\mathrm{H}$ patients answered positively and $64 \%$ of them answered 
negatively; $41 \%$ of the $\mathrm{O}$ patients answered positively and 59\% answered negatively. Overall, 37.5\% answered positively and $62.5 \%$ answered negatively.

Question no. 3 (Do you like your present voice?) A total of $63 \%$ of the $\mathrm{H}$ patients answered positively and $37 \%$ answered negatively; $59 \%$ of the $\mathrm{O}$ patients answered positively and $41 \%$ answered negatively. Overall, $61.5 \%$ answered positively and $38.5 \%$ answered negatively.

Question no. 4 (Would you consider a change in your voice tone?) A total of $36 \%$ of the $\mathrm{H}$ patients did not to perceive it as changed, whereas $64 \%$ of them did perceive it as changed; $31 \%$ of the $\mathrm{O}$ patients did not perceive a change, and $69 \%$ perceived a change. Overall, $65 \%$ of the patients referred to a changed voice, whereas $35 \%$ of them did not.

Question no. 5 (Did you undergo medical consultations for your voice?) A total of $8 \%$ of the $\mathrm{H}$ patients answered positively, whereas $92 \%$ of them answered negatively; $15 \%$ of the $\mathrm{O}$ patients answered positively, whereas $85 \%$ answered negatively; in total, 10\% answered positively and $90 \%$ answered negatively.
Question no. 6 (Did you undergo specialist consultations for your voice?) A total of $42 \%$ of the $\mathrm{H}$ patients answered positively and $58 \%$ of them answered negatively; $41 \%$ of the $\mathrm{O}$ patients answered positively, $59 \%$ answered negatively; in total, $42 \%$ were positive answers and $58 \%$ were negative answers.

Question no. 7 (Do you think a specialist consultation for your voice is necessary?) A total of $27 \%$ of the $\mathrm{H}$ patients answered positively and $73 \%$ answered negatively; $27 \%$ of the $\mathrm{O}$ patients answered positively and $73 \%$ answered negatively; in total, $27 \%$ answered positively and $73 \%$ answered negatively.

The most significant findings are summarized in Figure 1.

GRBAS scale: The GRBAS objective voice characteristics of group A, group B and group A plus B $(A+B)$ patients are shown in Table 1 . The voice was considered normal in $69 \%$ of groups $\mathrm{A}, \mathrm{B}$ and $\mathrm{A}+\mathrm{B}$; the most frequent abnormal modalities were "mildly rough" (group A: $10.5 \%$, group B: $13 \%$, group A + B: $11.5 \%$ ) and "mildly winded" (group A: $7.6 \%$, group B: $4.83 \%$, group A + B: 6.75\%; Table 1).

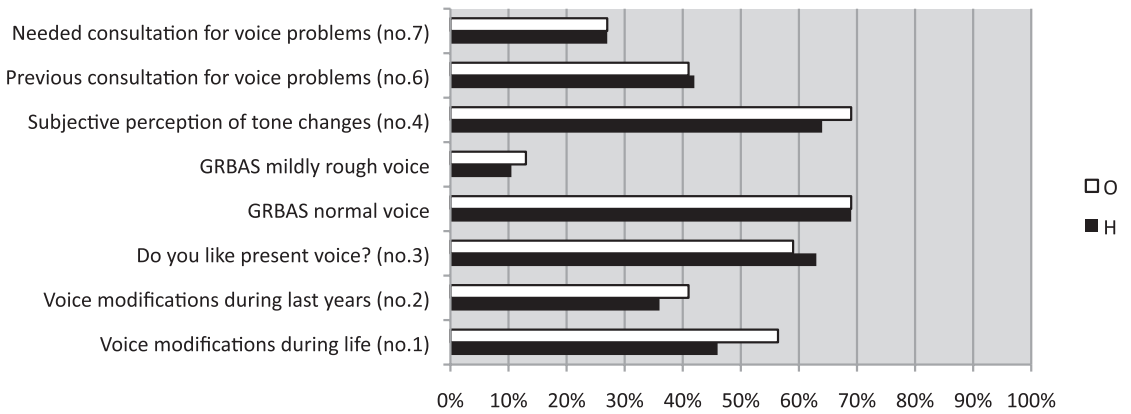

Figure 1 Percentage distribution of the main results of questionnaire 1 in the participants who had been hospitalized $(\mathrm{H})$ and the participants who underwent otorhinolaryngological consultations as outpatients $(\mathrm{O})$.

Table 1 Perceptive voice characteristics from the objective Grade, Roughness, Breathiness, Asthenia, Strain attributed to patients who had been hospitalized and patients who underwent otorhinolaryngological consultations as outpatients, and the two groups together

\begin{tabular}{lclc}
\hline GRBAS $(\%)$ & Group A & Group B & Group A + B \\
\hline Normal & 69 & 68.5 & 68.5 \\
Mildly rough & 10.5 & 13.1 & 11.5 \\
Moderately rough & 5.8 & 4.0 & 5.2 \\
Severely rough & 2.9 & 1.6 & 2.5 \\
Mildly winded & 7.6 & 4.8 & 6.7 \\
Moderately winded & 1.4 & 2.4 & 1.7 \\
Severely winded & 0.7 & 0.8 & 0.7 \\
Mildly strained & 2.5 & 2.4 & 2.5 \\
Moderately strained & 0 & 0.8 & 0.2 \\
Severely strained & 0 & 0.8 & 0.2 \\
Asthenic & 0 & 0 & 0
\end{tabular}

Group A consisted of patients who had been hospitalized. Group B consisted of patients who underwent otorhinolaryngological consultations as outpatients. GRBAS, Grade, Roughness, Breathiness, Asthenia, Strain. 


\section{Questionnaire VHI}

The partial and total VHI scores in the three groups are shown in Table 2 .

\section{Association between questions no. 1-5 and no. 2-5 of questionnaire 1}

A strong correlation between the perception of past and present voice modifications with specialist consultation was found in all the $\mathrm{H}$ and female patients (0.007).

\section{Association between questions no. 1-7 and no. 2-7 of questionnaire 2}

A significant correlation between past and present voice modifications with the opportunity to undergo specialist consultation was found in all the $\mathrm{H}$ (group A) patients, and in both men and women (0.000; 0.000 and 0.003). Among the $\mathrm{O}$ population, only the correlation between past voice modifications and the opportunity to undergo specialist consultation was statistically significant.

\section{Association between question no. 3 of questionnaire 1 and GRBAS}

A correlation between acceptance of one's own voice and the objective evaluation was significant in all the male and female $\mathrm{H}$ patients (0.000), as well as in all the male $\mathrm{O}$ patients.

\section{Association between question no. 4 of questionnaire 1 and pathological GRBAS (subjective perception of changed tone)}

No significant correlation was found, either in the $\mathrm{H}$ or in the $\mathrm{O}$ population group.

\section{Correlation between VHI and question no. 7 of} questionnaire 1

The correlation between severe phonatory handicaps and the need for specialist consultation was strong in all (point-biserial correlation [rpb] 0.66), male (rpb 0.51) and female (rpb 0.72) O patients; and in all (rpb 0.36), male (rpb 0.40) and female (rpb 0.28) H patients $(P<0.0001)$.

\section{Correlation between VHI and GRBAS}

The correlation between severe phonatory handicaps and the objective evaluation of changed tone was strong $(P<0.0001)$ in all (rpb 0.46), both male (rpb 0.48) and female (rpb 0.42) $\mathrm{H}$ patients; and in all (rpb 0.35; $P<0.0001$ ), male (rpb 0.50; $P=0.003$ ) and female (rpb $0.30 ; P=0.008) \mathrm{O}$ patients.

\section{Subjective auditory evaluation}

\section{Questionnaire Hearing Quality}

Question no. 1 (Did your hearing change during your life?) A total of $58 \%$ of the $\mathrm{H}$ patients answered yes and $42 \%$ answered no; $71 \%$ of the $O$ patients answered yes and $29 \%$ of them answered no. Overall, $62 \%$ answered yes and $38 \%$ answered no.

Question no. 2 (Has your hearing changed during recent years?) A total of $58 \%$ of the $\mathrm{H}$ patients answered yes and $42 \%$ answered no. Among $\mathrm{O}$ patients, $71 \%$ answered yes and 29\% answered no. Overall, 62\% answered yes and 38\% answered no.

Question no. 3 (Did you undergo an audiological test?) A total of $43 \%$ of the $\mathrm{H}$ patients answered yes and

Table 2 Voice Handicap Index (VHI). Partial (Physical, Emotional and Functional) and total scores in the three study groups (A: hospitalized patients; B: outpatients; $\mathrm{A}+\mathrm{B}$ )

\begin{tabular}{lccc}
\hline & Group A & Group B & Group A + B \\
\hline P (physical) & 1040 & 875 & 1915 \\
Mean (SD) & $3.77(5.34)$ & $7.06(8.23)$ & $4.78(6.54)$ \\
Min & 0 & 0 & 0 \\
Max & 28 & 34 & 34 \\
E (emotional) & 448 & 490 & 938 \\
Mean (SD) & $1.63(3.97)$ & $3.95(6.35)$ & $2.34(4.97)$ \\
Min & 0 & 0 & 0 \\
Max & 24 & 24 & 26 \\
F (functional) & 754 & 593 & 1347 \\
Mean (SD) & $2.74(4.81)$ & $4.78(6.53)$ & $3.36(5.47)$ \\
Min & 0 & 0 & 0 \\
Max & 27 & 23 & 27 \\
Total & 2244 & 1958 & 4202 \\
\hline
\end{tabular}

Group A consisted of patients who had been hospitalized. Group B consisted of patients who underwent otorhinolaryngological consultations as outpatients. 
57\% answered no. Among $\mathrm{O}$ patients, 56\% answered yes and $44 \%$ answered no, with overall responses of $47 \%$ yes and $53 \%$ no.

Question no. 4 (Have you been examined by an audiologist) A total of $56 \%$ of the $\mathrm{H}$ patients answered yes and 44\% answered no; among $\mathrm{O}$ patients, $69 \%$ answered yes and $31 \%$ answered no, with overall responses of $60 \%$ yes and $40 \%$ no.

Question no. 5 (Do you think you are in need of an audiological examination for your hearing?) A total of $46 \%$ of the $\mathrm{H}$ patients answered yes and $54 \%$ answered no; among $\mathrm{O}$ patients, $65 \%$ answered yes and $35 \%$ answered no, with overall responses of $51.5 \%$ yes and $48.5 \%$ no.

Question no. 6 (Are there any cases of hearing loss in your family?) A total of $26 \%$ of the $\mathrm{H}$ patients answered yes and $74 \%$ answered no; among the $\mathrm{O}$ patients, $28 \%$ answered yes and $72 \%$ answered no, with overall responses of $27 \%$ yes and $73 \%$ no.

Question no. 7 (Did you ever suffer an ear infection?) A total of $22 \%$ of the $\mathrm{H}$ patients answered yes and $78 \%$ answered no; among the $\mathrm{O}$ patient population, $31 \%$ answered yes and $69 \%$ answered no, with overall responses of $24.5 \%$ yes and $75.5 \%$ no.

Question no. 8 (Do you have noise in the ears?) A total of $33 \%$ of the $\mathrm{H}$ patients answered yes and $67 \%$ answered no; among the $\mathrm{O}$ patients, $38 \%$ answered yes and $62 \%$ answered no, with overall responses of $35 \%$ yes and $65 \%$ no.

Question no. 9 (Do you suffer from vertigo?) A total of $36 \%$ of the $\mathrm{H}$ patients answered yes and $74 \%$ answered no; among the $\mathrm{O}$ patients, 39\% answered yes and $61 \%$ answered no, with overall responses of $30 \%$ yes and $70 \%$ no.

The most significant findings are summarized in Figure 2.
Anonymous questionnaire on the subjective evaluation of verbal-acoustic communication $S A C(V)$

Partial (handicap and disability) and total results for groups A, B and A + B are reported in Table 3 . The mean handicap perception in all the cases was 3 , with that of disability measuring 3.97 and the mean handicap plus disability measuring 7.18.

Association between questions no. 1, 2, 8 and 9, and questions no. 3 and 4 (questionnaire 2) Correlation between changes in past and present hearing and prior audiological consultation. In the $\mathrm{H}$ patients, all the correlations were significant $(0,000)$; among the $\mathrm{O}$ patients, only females showed significant correlations of all the items $(0.000-0.064)$, except for that between questions no. 8 and 4 , and questions no. 9 and 4 . In male $\mathrm{O}$ patients, the latter was significant $(0.045)$. In all the $\mathrm{O}$ patients, only the correlations between questions no. 1 and $3(0.001)$, and between no. 8 and $3(0.075)$ were statistically significant.

Association between questions no. 1-5, 2-5, 8-5 and 9-5 of questionnaire 2 Correlation of auditory symptoms and the need to undergo an audiological consultation. In the $\mathrm{H}$ patients, in men and women, all the correlations were significant except for 9-5. Additionally, all the male and female $\mathrm{O}$ patients showed a significant correlation (0.000) except for no. 9-5.

Association between questions no. 1, 2, 8 and 9, and questions no. 6 and 7 of questionnaire 2 Correlation between auditory symptoms and familiarity with hearing disorders ${ }^{6}$ and prior infections. ${ }^{7}$ This correlation was significant $(0.004-0.000)$ in all the $\mathrm{H}$ patients and in the female patients $(0.014-0.000)$. The correlations between auditory symptoms and prior ear infections in all the $\mathrm{H}$ patients $(0.02-0.002)$ and in the male patients (0.008-0.027) were also significant.

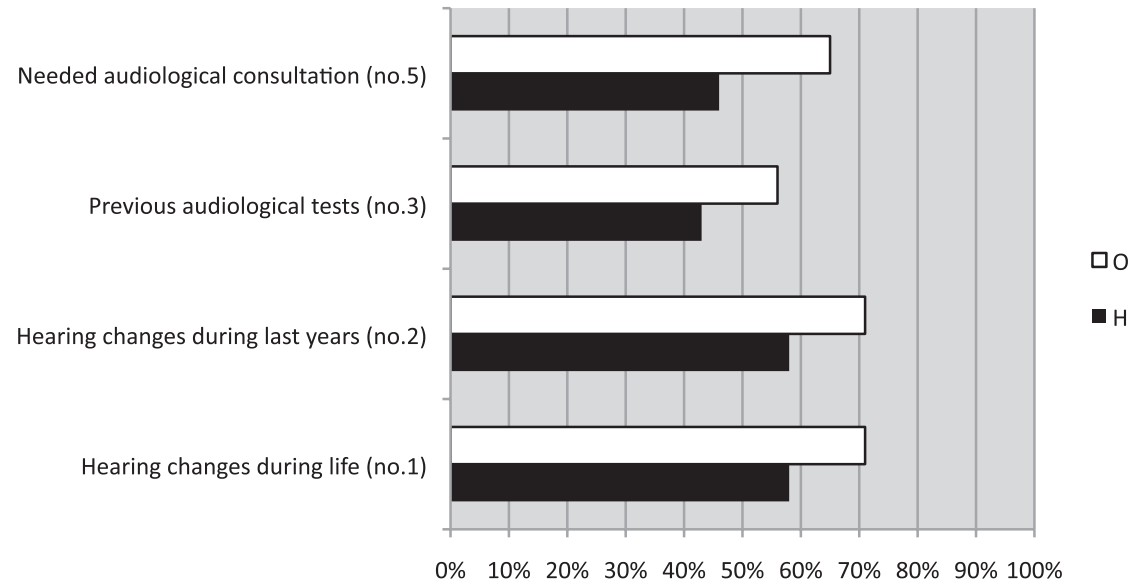

Figure 2 Percentage distribution of the main results of questionnaire 2 in participants who had been hospitalized $(\mathrm{H})$ and the participants who underwent otorhinolaryngological consultations as outpatients $(\mathrm{O})$. 
Table 3 Self-Assessment of Communication (SAC) as handicap, disability and total scores in the three study groups (A: hospitalized patients, B: outpatients, A + B)

\begin{tabular}{lccc}
\hline & Group A & Group B & Group A + B \\
\hline Handicap & 781 & 500 & 1281 \\
Mean (SD) & $2.82(3.85)$ & $4.03(4.52)$ & $3.02(4.1)$ \\
Min & 0 & 0 & 0 \\
Max & 15 & 16 & 16 \\
Disability & 1017 & 574 & 1591 \\
Mean (SD) & $3.68(5.15)$ & $4.62(5.48)$ & $3.97(5.27)$ \\
Min & 0 & 0 & 0 \\
Max & 20 & 20 & 20 \\
Total & 1798 & 1074 & 2872 \\
Mean (SD) & $6.51(8.72)$ & $8.66(9.75)$ & $7.18(9.09)$ \\
Min & 0 & 0 & 0 \\
Max & 34 & 36 & 36 \\
\hline
\end{tabular}

Group A consisted of patients who had been hospitalized. Group B consisted of patients who underwent otorhinolaryngological consultations as outpatients.

Correlation between SAC and question no. 5 of questionnaire 2 Correlation between a high score on the SAC questionnaire and requests for specialist consultations. High significance was found in all the $\mathrm{O}$ and $\mathrm{H}$ patients, both men and women $(P<0.0001)$ with $\mathrm{rpb}=0.55$ (all $\mathrm{O}), \mathrm{rpb}=0.58$ (male $\mathrm{O}), \mathrm{rpb}=0.53$ $($ female $\mathrm{O}) ; \mathrm{rpb}=0.52($ all $\mathrm{H}), \mathrm{rpb}=0.47($ male $\mathrm{H})$ and $\mathrm{rpb}=0.60$ (female $\mathrm{H}$ ).

\section{Discussion}

Age-related changes in the voice production organs and sensorineural hearing structures produce voice and hearing modifications in a considerable part of the geriatric population, with eventual negative impacts on daily communication and life. If one considers that these voice and hearing disturbances might be concomitant with other effects that could have been developing throughout life, it seems extremely important to identify them during earlier stages to apply appropriate treatment.

Psychometric studies on the perception of phonatory handicaps (VHI) and on the functional impact of vocal disorders in daily life (Voice-Related Quality of Life) have shown that the elderly population seems not to be very inclined to self-declare voice changes, because they consider these changes to be normal for their age. Should they perceive it, furthermore, a prevalent physical handicap is indicated that would not affect either the social quality of life or the patient's general health. ${ }^{10,12}$

The present study aimed to evaluate how a geriatric population would perceive voice and/or hearing changes, weighing the relative handicaps, what types of perceived handicaps prevailed, and the correlation between the perceived handicap and request for treat- ment. Furthermore, by using different open and standard questionnaires regarding the voice, the correlation between the subjective evaluation of voice impairment and requests for specialist consultations were analyzed. Two geriatric populations were considered in the present study, assuming that some differences between outpatients and hospitalized patients might exist, the latter being more likely to retain poorer performances as a result of impaired health conditions that required hospitalization. To avoid obtaining biased data, both of the populations under study were selected for not being primarily treated for hearing or voice problems, or for being admitted to otolaryngological wards.

The outcomes of the subjective, anonymous questionnaire regarding age-related changes in voice showed that nearly half of the participants reported voice modifications during their entire lives, but with only a smaller percentage of them referring to voice modifications in recent years. Furthermore, the majority of the patients liked their own voices, although they realized that its tone had changed $(64 \%)$, with a prevalent hoarse character $(26 \%)$. No differences regarding the anonymous voice questionnaire were found between the two study groups $\mathrm{H}$ and $\mathrm{O}$. The objective GRBAS evaluation showed a high percentage of participants with normal voices $(69 \%)$, whereas voice alterations were found in the remaining percentages and hoarseness in $61 \%$ of this remainder. Hence, the subjective and the objective evaluations were in agreement regarding changes in voice production, with most reporting hoarseness in tonality. Despite this evidence, it is quite surprising that the majority of the patients in both groups never underwent specialist consultations before for voice problems, although most of them thought they required it. Nonetheless, among the hospitalized patients of both sexes 
who received specialist consultations, a significant correlation was found with the need to plan further treatment.

Although the subjective and the objective evaluations were strongly correlated in patients of both groups with normal voices, when a voice impairment was identified, it was poorly correlated with the patient's subjective perception. In addition, the GRBAS protocol was significantly correlated with the acceptance of one's own voice (correlation between question no. 4 and GBRAS) in both groups, confirming its great value for the assessment of the subjective perception of vocal impairment. From these findings, it would be possible to assume that the perception of a change in voice mostly relies on tone variations and not on the difference between pathological and normal tones. In this regard, the best question for revealing this perception was "Do you like your voice?".

All the patients showed low scores for partial and total voice handicaps on the VHI. In this regard, only the physical scale reached the maximum score of 34 in groups $\mathrm{O}$ and 28 in group $\mathrm{H}$, showing that, in accordance with the literature ${ }^{7,15,16}$ and also in the present study, the perceived handicap of voice was mainly physical in typology. Furthermore, the $\mathrm{O}$ patients seemed to perceive the handicap more than the $\mathrm{H}$ patients, presumably as the latter were more conditioned by the main pathology that caused the admittance. Despite the rather low incidence of handicap perception, the latter was strongly correlated in both groups and sexes with objective evaluations of an impaired quality of voice. This finding shows that when the elderly subjects display a voice-related handicap, this impairment is effectively present. The subjective perception of voice alteration and its relative handicap will also motivate requests for treatment in both sexes, as shown by the significant correlation between VHI and question no. 6 , relative to the need to undergo a specialist evaluation in the future.

When considering hearing function, it would be likely to assume that the use of questionnaires for the identification of the perception of a hearing disability might be useful for selecting, among the elderly population, who needs a hearing assessment and eventual rehabilitation with a hearing aid. In this regard, the majority of the participants in both groups referred to hearing changes during their life, particularly in recent years, that was strongly correlated with a previous audiological examination or the need for an audiological examination. The subjective perception in hearing changes, in terms of handicap and disability (SAC), showed very low mean values in both groups, both for handicap (maximum score of 15 in group $\mathrm{H}$ and 16 in group $\mathrm{O}$ ) and for disability (maximum score of 20 , in both groups). These values, although low, were significantly correlated with care request in both sexes (correlation between SAC and question no. 5 of questionnaire 2).
Hearing modification was, particularly in the $\mathrm{H}$ patients, significantly correlated with concomitant auditory symptoms, such as tinnitus, as well as with earlier or future planning to undergo a specialist consultation. The presence of auditory symptoms was furthermore significantly correlated with a positive family history, as well as with previous auricular infections. Because this association was found in $46 \%$ of the participants, one could assume that in nearly half of the elderly population, presbycusis represents a real issue..$^{3,7,17,18}$

The present results also showed that when a handicap or disability was assessed as a perceived impairment relative to impairment in verbal-acoustic communication, with subjective evaluation of recently occurring dysfunctions, low perception scores were recorded. When, instead, the impairment per se was evaluated along with its input for a request of diagnosis and treatment, the phonatory impairment, although present, was underestimated by elderly participants, who were unable to distinguish between "age-normal" and "pathological". In hearing, things are not going in the same manner, because hearing loss has a much greater impact on communication impairment, although the appropriate rehabilitation with hearing aids appears not yet to have been applied extensively. For voice impairments, it could be appropriate to carry out in all elderly patients a diagnostic workout by using fiberoptic dynamic evaluation of the larynx and voice, and to select those patients who might require a medical or surgical treatment, or rather a voice rehabilitation program in the case of dysfunctional pathologies.

The clinical approach to voice and hearing impairment in the elderly needs to be assessed through systematic specialist consultations, because these symptoms are rarely spontaneously referred by patients who also tend to consider them as non-pathological issues. Yet, these impairments might likely be inducing physical, functional and psychosocial problems that could negatively affect the quality of relational and general life. Appropriate rehabilitative protocols, including a wider use of hearing aids for the commonly observed hearing impairment, are therefore suggested to minimize the negative impact on the whole communication system of this age population.

\section{Disclosure statement}

The authors declare no conflict of interest.

\section{References}

1 Plank C, Schneider S, Eysholdt U, Schutzenberger A, Rosanowski F. Voice and health-related quality of life in the elderly. J Voice 2011; 25: 265-268. 
2 Golub JS, Po-Hung C, Otto KJ, Hapner E, Jhons MM. Prevalence of perceived dysphonia in a geriatric population. J Am Geriatr Soc 2006; 54: 1736-1739.

3 Qi H, Tang J. Age-related hearing loss or presbycusis. Eur Arch Otorhinolaryngol 2010; 267: 1179-1191.

4 Costa HO, Matias C. Vocal impact on quality of life of elderly female subjects. Rev Bras Otorinolaringol 2005; 71: 172-178.

5 Salomon G, Vesterager V, Jagd M. Age-related hearing difficulties. Audiology 1988; 27: 164-178.

6 Schow RL, Nerbonne MA. Communication screening profile: use with elderly clients. Ear Hear 1982; 3: 135-147.

7 Jacobson BH, Johnson A, Grywalsky C et al. The Voice Handicap Index (VHI): development and validation 1997. Am J Speech Lang Pathol 1997; 6: 66-70.

8 Ventry I, Weinstein B. The hearing handicap inventory for the elderly: a new tool. Ear Hear 1982; 3: 128-134.

9 Hogikyan ND, Sethuraman G. Validation of an instrument to measure Voice Related Quality of Life (V-RQoL). J Voice 1999; 13: 557-569.

10 Schneider S, Plank C, Eysholdt U, Schutzenberger A. Voice function and voice-related quality of life in the elderly. Gerontology 2011; 57: 1-6.

11 Tajvar M, Arab M, Montazeri A. Determinants of healthrelated quality of life in elderly in Tehran, Iran. BMC Public Med 2008; 8: 109-114.

12 Vuorialho A, Karinen P, Sorri M. Effect of hearing disability and quality of life in the elderly. Int J Audiol 2006; 45: 400-405.

13 Murry T, Medrado R, Hogikyan ND, Aviv JE. The relationship between ratings of voice quality and quality of life measures. J Voice 2004; 18: 183-192.

14 Hirano M. Clinical Examination of Voice. New York: Springer Verlag, 1981; 81-84.
15 Portone CR, Hapner ER, McGregor I, Otto K, Johns IIIMM. Correlation of the voice handicap Index (VHI) and the Voice-Related Quality of Life Measure (VRQoL). $J$ Voice 2007; 21: 723-727.

16 Rosen CA, Lee AS, Osborne J, Zullo T, Murry T. Development and validation of the voice handicap index- 10 . Laryngoscope 2004; 114: 1549-1556.

17 Scholtz A, Kammen-Jolly K, Felder E, Hussl B. Selective aspects of human pathology in high-tone hearing loss of the aging inner ear. Hear Res 2001; 157: 77-86.

18 Seidman M, Ahmad N, Bai U. Molecular mechanisms of age-related hearing loss. Ageing Res Rev 2002; 1: 331-343.

\section{Supporting information}

Additional Supporting Information may be found in the online version of this article at the publisher's web-site:

Appendix S1 Information form.

Appendix S2 Form for collection of general clinical information.

Appendix S3 Anonymous subjective questionnaire on senile modifications of the voice.

Appendix S4 Anonymous subjective questionnaire on verbal-acoustic communication.

Appendix S5 Voice Handicap Index (VHI).

Appendix S6 Self Assessment of Communication (SAC).

Appendix S7 Grade, Roughness, Breathiness, Asthenia, Strain (GRBAS). 